\title{
Distributed Swarm Optimization Modeling for Waste Collection Vehicle Routing Problem
}

\author{
ELGAREJ Mouhcine, MANSOURI Khalifa, YOUSSFI Mohamed, BENMOUSSA Nezha, EL FAZAZI Hanae \\ Laboratory SSDIA, ENSET \\ University Hassan II \\ Mohammedia, Morocco
}

\begin{abstract}
In this paper, we consider a complex garbage collection problem, where the residents of a particular area dispose of recyclable garbage, which is collected and managed using a fleet of trucks with different weight capacities and volume. This tour is characterized by a set of constraints such as the maximum tour duration (in term of distance and the timing) consumed to collect wastes from several locations. This problem is modeled as a garbage collection vehicle routing problem, which aims to minimize the cost of traveling routes (minimizing the distance traveled) by finding optimal routes for vehicles such that all waste bins are emptied and the waste is driven towards the disposal locations. We propose a distributed technique based on the Ant Colony system Algorithm to find optimal routes that help vehicles to visit all the wastes bins using interactive agents consumed based on the behavior of real ants. The designed solution will try to create a set of layers to control and manage the waste collection, each layer will be handled by an intelligent agent which is characterized by a specific behavior, in this architecture a set of behaviors have been designed to optimizing routes and control the real time capacity of vehicles. Finally, manage the traffic messages between the different agents to select the best solutions that will be assigned to each vehicle. The developed solution performs well compared to the traditional solution on small cases.
\end{abstract}

Keywords-Vehicle routing system; ant colony optimization; multi-agent system; garbage collection system

\section{INTRODUCTION}

The vehicle routing problem [1]-[3] includes the optimization of a set of minimum cost transportation routes to serve a various set of customers using a dynamic or a fixed fleet of transportation trucks (vehicles, trucks ...). The map of this problem contains a set of routes associated with several points or locations named as depots. Each customer is visited by only one vehicle. The vehicle must follow the optimal route proposed by the system. This problem combines several types of constraints, such as the limit of the total distance covered by each vehicle and total working time per day, the availability of resources (vehicles, customers' data, salesman ...).

There are many different models of vehicle routing problem. We consider in this paper a waste collection problem [4]-[8] where a number of vehicles are used for collecting waste from different households (clients). Those wastes must be collected from several areas and take them back toward the disposal facilities locations. Each vehicle is characterized by its capacity and the number of waste locations to visit.
At the beginning, all available vehicles are assigned to the depot (Fig. 1). Each vehicle will start its cycle to collect wastes from the different locations according to the path planning proposed by the central unit. Wastes are collected until the capacity of the vehicle is reached. Then it disposes the waste to a disposal facility predefined and repeats the same process during its working time. At the end, all the vehicles must return to the depot.

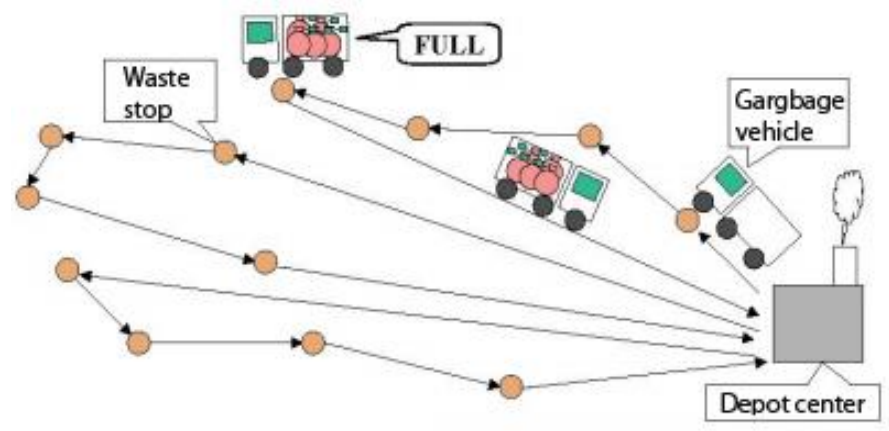

Fig. 1. The garbage collection management.

In the classical garbage collection method, the system is based on a successive process, it means that when a vehicle is not able to accomplish his task we cannot use another vehicle to terminate the unfinished tasks, this happens when a vehicle exceeds his capacity limit. In the other hand, if we increase the number of available vehicles to avoid the capacity limit and to reach all the wastes points, it may produce that vehicles will use only the three quarters of their capacity for collecting all wastes from the different locations. Actually, with the new intelligent systems, we can create a new distributed mechanism to control the map of the waste locations and the disposal facilities to built a new path planning for each vehicle based on real time data sent by the vehicle which contains his actual position and his capacity. According to this information, the system will be able to assign the best circuit to the nearest vehicle. Actually, we can use a set of embedded systems [12], [14] for collecting all helpful data such as the actual capacity of each vehicle at each location, also, notifying each vehicle by the new path planning created when one of the available vehicles arrive at his capacity limit. We can say that our problem is based on dynamic data, so, the system should be able to receive the new information and re-used to recalculated the new alternative paths planning for each working vehicle. 
The main objective is to propose a distributed environment based on multi-agent entities to create a parallel system to solve the garbage collection problem using the ACS (Ant colony System) algorithm [23] to compute the best route for every vehicle and control the different entities that collaborate together for constructing and monitoring the process of waste collection. The system is based on three main layers, each one is controlled by an intelligent agents which are characterized by a specific behavior, we use agents to collect real time information about the state of the vehicle capacity, other agents have been created to travel between the set of bins and find the optimal routes which minimize the total distance covered by each vehicle and a controller agent is defined to control and manage the traffic of communication between the set of agents and supervising the state of vehicles when some trucks arrive at their capacity limit this agent will be able to create alternatives routes for the rest of trucks with the collaboration of the others agents. The results showed that the distributed technique is able to control the waste collection and find good results compared with the classical methods for solving the garbage collection system.

The outline of this work is as follows. In the next section, we define related works done to solve this type of problems. In Section 3, we describe the problem of waste collection and how can be modeled as vehicle routing problem. The technique used for solving the waste collection problem is proposed in Section 4 that aims to find the optimal routes for vehicles. The distributed solution using multi-agent system is defined in Section 5. The computational results are described in Section 6. Finally, the conclusion is given in the last section.

\section{PREVIOUS WORKS}

Waste management has become a major problem in all the part of the world and tends to grow day by day. In what follows some works related to this problem are cited.

In [11], the authors described a new solution for the optimization garbage removal problem in the large. This work provides a system architecture that helps find the time-optimal dynamic route for garbage trucks within the "Smart Clean City" project. They proposed a formal mathematical model of the task of a dynamic optimal route and formal the optimization criterion for time-optimal garbage collection of all waste from landfills.

A new solution has been proposed in [12] for solving and finding the most efficient path planning to collect solid waste based on the Particle swarm optimization method (PSO) and the traveling salesman problem (TSP) that shows a very important result for finding the optimal routes toward the wastes locations. Further, to find the locations of wastes, their proposed method is based on two main technologies are GSM/GPRS and ZigBee.

Moreover, authors in [14] presented a novel model of waste bin monitoring system based on wireless sensor network in order to minimize the operation costs and help the system create optimal routes based on the data collected from the different sensors. This proposed model is based on a set of sensors to measure the amount of wastes at each stop and for the GPS location using ZigBee, allowing by this information the system to better control the collection process. Their model is composed of three layers such as lower, upper and middle tier. The lower structure contains bin with sensor node to measure and send real time status to the next tier, the middle line stores and transmits bin data to the upper tier that stores and analyze the received data for further practicing.

Recently, a new IoT based waste collection method is proposed in [13] to automate the waste identification, localization and collection process. Also, they implemented an optimized and automated garbage collection system with the use of a vast sensor network able of collecting waste data. They introduced a distributed architecture using an optimizing algorithm to control the next step of collecting wastes from various locations, in order to manage the waste collection process.

\section{PROBLEM DEFINITION}

The formulation of the given problem can be viewed as an interconnected graph $\mathrm{G}=\left(V_{w}, V_{f}, \mathrm{~A}\right)$ where $V_{w}=\left\{v_{1}, v_{2}, v_{3}, v_{4}, \ldots v_{n}\right\} \quad$ is the wastes locations, $V_{f}=$ $\left\{v_{1}, v_{2}, \ldots v_{m}\right\}$ is a set of disposal facilities, including the start depot.

The parameter $A=\left\{(i, j): v_{i}, v_{j} \in V_{w} \cup V_{f}\right\}$ is the route between two connected nodes $\left(N_{i}, N_{j}\right)$, and a cost $d_{i j}$ (distance) is associated with the route $\left(N_{i}, N_{j}\right)$.A fleet of m vehicles with various capacity is available. A given route will contain a set of waste locations and intermediate facilities.

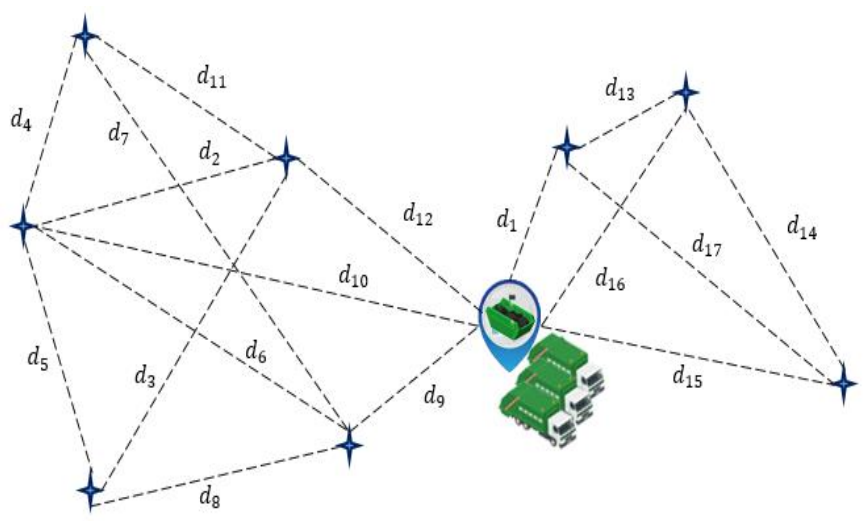

Fig. 2. Vehicle routing for wastes collections system.

Each vehicle leaves the depot and starts visiting the wastes from the given locations (Fig. 2). When a vehicle meets his maximum capacity, it reaches an intermediate facility for the unloading operation, then it starts again his trip from the last visited point and repeats the same process or we assign his task to the nearest vehicle to complete the unfinished task. When all waste locations are visited or all vehicles finished their tasks, the available fleet of vehicles will return to the garage.

The problem consists of determining the optimal path planning for each vehicle by considering the following constraints:

- Every waste location is visited once and only by one vehicle. 
- Every route starts from the depot and cross several stops and intermediate facilities.

- Not exceed the capacity of vehicles.

- Optimizing the total distance traveled by each vehicle.

- Increase the quantity of wastes transported by vehicles.

- Produce alternatives paths planning when one of the vehicles meets his capacity limit before visiting all their wastes locations.

\section{ANT COLONY OPTIMIZATION FOR THE WASTE COLLECTION SYSTEM}

The Ant Colony Optimization (ACO) technique presented in [9], [10], is a meta heuristic technique where a set of artificial ants are used to solve several optimization problems. ACO technique has been used to solve hard problem such as Traveling Salesmen problem (TSP) [17]-[19], Vehicle Routing Problem [1]-[3], etc.

ACO is based on the behavior of the ants in the real world. In reality, the ant is able to find the shortest path between a food source and the nest. In parallel, they can sense the change in the space of search and act accordingly based on the information shared between ants using a volatile chemical substance called pheromones. An ant which has the ability to deposit pheromone on the path has also the possibility to measure the amount of pheromone deposited by the others ants. It always chooses to move towards the higher density of pheromones. When several ants choose the same path it means that the concentration of pheromone on this path will increase accordingly to the amount of pheromone deposited by those ants. As a result, ants will be able to follow the optimal path produced and followed by the majority of ants.

In reality, the behavior of the ACO techniques is based on two main steps to select and compute the optimal path for a vehicle routing problem: 1) the route construction; 2) the pheromone trail update.

\section{A. The Route Construction}

The present ACS (Ant Colony system) algorithm for the garbage collection starts with a group of ants, which contains $n$ individual ants. These ants work together in parallel to construct routes. Each ant will construct a complete tour for the given map of waste locations. The group of vehicles will be simultaneously dispatched between the constructed routes. The process of route construction can be described as follows: Initially, each artificial ant (an intelligent developed agent) starts at the depot and try to select the next waste location to visit from the list of available locations and the storage capacity of the vehicle is updated before a new location is selected. When the capacity of the vehicle is met, then the system will restart the process of collection and try to prepare a new distributed path planning for the available vehicles using the new map of wastes locations.

In the new improved version of the ACO for the garbage collection system, each worker ant needs to create a vehicle path planning (routes) that visits many wastes stops. Each ant select the next node to visit using a probabilistic transition [22] showed as follows (1):

$$
p_{i}^{k}=\left\{\begin{aligned}
\frac{\left[\tau_{i j}\right]^{\alpha}\left[\eta_{i j}\right]^{\beta}}{\sum_{j \in N_{i}^{k}}\left[\tau_{i j}\right]^{\alpha}\left[\eta_{i j}\right]^{\beta}}, & \text { if } j \in N_{i}^{k} \\
0, & \text { otherwise }
\end{aligned}\right.
$$

Where $\tau_{i j}$ is the pheromone density on the route between the current stop $\mathrm{i}$ and possible node $\mathrm{j} . \eta_{i j}$ is the visibility of edge $(\mathrm{i}, \mathrm{j})$. Here we define $\eta_{i j}$ as a heuristic input value, included to control the convergence of the system toward the optimal solutions depending on what the user needs to get at the end of the process. Thus, $\alpha$ and $\beta$ the relative influence of the visibility values and the pheromone trails. $N_{i}^{k}$ is the set of waste locations which have not been visited.

\section{B. Pheromone Trail Updating}

This step is divided into two main parts: a local and a global update [22]. The local update is performed through routes construction and done by each ant during his travel, the global update of pheromone is performed when all ants arrives at their end or when they finished their tours, the system will be able to update all routes by adding a new amount of pheromone. The following rule (2) describes the process of the local update:

$$
\tau_{i j}^{n e w}=(1-\boldsymbol{\rho}) \tau_{i j}^{o l d}+\Delta \tau_{i j}
$$

Where $0<\boldsymbol{\rho}<1$ is a local pheromone decay parameter, $\tau_{0}=1 / n C^{n n}$ is the pheromone value deposited on $\operatorname{arc}(\mathrm{i}, \mathrm{j})$, where $\mathrm{n}$ is the number of locations and $C^{n n}$ is the length of the initial solution proposed by a stochastic nearest neighbor heuristic technique.

On the other hand, global updating is used to increase the search in the neighborhood of the best and optimal solution computed. In ACS algorithm, only the best solution is allowed to apply the global update of pheromone. Global updating of trail is performed according to the following rule (3):

$$
\begin{gathered}
\tau^{\text {new }}{ }_{i j}=(1-\boldsymbol{\rho}) \tau^{\text {old }}{ }_{i j}+\Delta \tau_{i j}+\Delta \tau^{\text {best }}{ }_{i j} \\
\Delta \tau^{\text {best }}{ }_{i j}=>\sum_{\mathrm{k}=1}^{m} \Delta \tau_{i j}{ }^{k}=\left\{\begin{array}{cl}
\frac{Q}{L^{\text {best }},}, & \text { if edge ij used in the best path } \\
0, & \text { else }
\end{array}\right. \\
\text { V. DISTRIBUTED ARTIFICIAL ANTS FOR SOLVING THE } \\
\text { GARBAGE COLLECTION PROBLEM USING MULTI-AGENT } \\
\text { SYSTEM }
\end{gathered}
$$

In this paper, a distributed environment based on multiagent entities is proposed to create a parallel system to solve the garbage collection problem using the ACS (Ant colony System) algorithm to compute the best route for every vehicle and control the different entities that collaborate together for constructing and monitoring the process of evolution toward the optimal path planning for a fleet of vehicles (Fig. 3).

In the proposed approach, we firstly, design a new distributed system to control the process of communication between agents. Secondly, a decentralized system is used to manage the convergence of the system toward the best solution 
(optimal routes that will help vehicles to visit all wastes locations and terminate their tasks). Finally, we implement a parallel version of the ACS algorithm for finding the optimal solution based on artificial ants created only for this work.

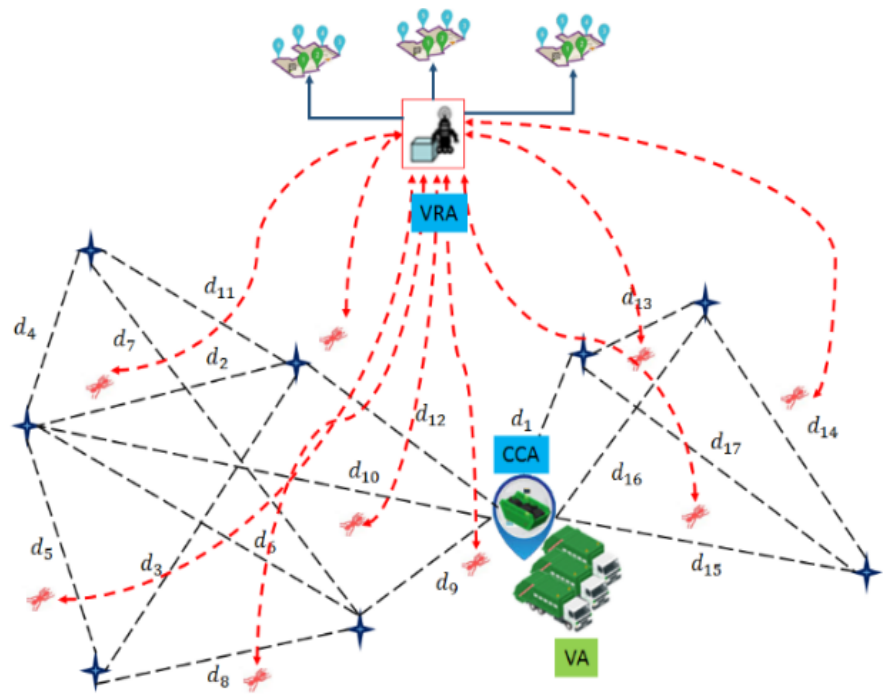

Fig. 3. Distributed architecture for solving waste collection using multiagent system.

Various agents were developed to create the distributed environment, which holds intelligent entities. The first one is the Central command Agent (CCA) and its function is to interact with the user and the different agents that cooperate together for solving and finding alternatives solutions as running time. The CCA will allow the user to draw its own graph, which contains a set of waste locations and the different routes that link those nodes. In the same line, the CCA will control the process of waste collection and observe the capacity of vehicles in real time to avoid the overflow of the capacity, also, communicate with external agents to create alternative routes in the case of exceeding the capacity limit.

The second agent is the Vehicle Agent (VA) that helps for controlling the capacity state of the vehicle and remain the CCA with his actual location (the current visited waste stop). When the vehicle meets his capacity limit, the VA will notify the CCA with this case and wait for the new instructions to follow.

Another artificial component is the Vehicle Routing Agent (VRA), which contains a set of artificial ants that work in parallel based on the same behavior of real ant for finding the optimal routes in the given map by respecting the different constraints that characterize the problem. In Fig. 3, we describe our solution used to solve the garbage collection problem.

\section{A. Central Command Agent (CCA)}

In the proposed architecture, the CCA will monitor the search space which is viewed as a map made from several wastes locations, each one is identified by his latitude and longitude and the routes between those points is drawn by a user interface dedicated to construct the map of the problem. All the available vehicles are identified by the CCA through the VA, this CCA will exchange several messages with those agents such as the path planning that will be followed and used by this VA, also, the VA must send a set of alerts which remain the CCA with the current capacity of the vehicle, in the case of the capacity overflow limit, the VA will inform the CCA and this one will ask the VRA for new alternative actions to avoid this situation and to create new routes for each available vehicle. It is worth noting without forgot that each vehicle should start his tour from the last visited stop and continue his path planning to finish his tour.

Based on the proposed model, the depot is viewed as the center of our map (Fig. 4), each vehicle should start from the depot and picked up wastes from the different locations and take them to the nearest disposal facilities then return to the depot at the end of their tours. The CCA manage a graph with a set of nodes (i.e. depot, wastes locations, disposal facilities and vehicles) and edges that define the routes between two adjacent locations. The cost of every edge is marked by the distance between two nodes. At the beginning, the CCA will initialize the parameters of the problem by generating a graph that contains the different components of the problem (Fig. 4).

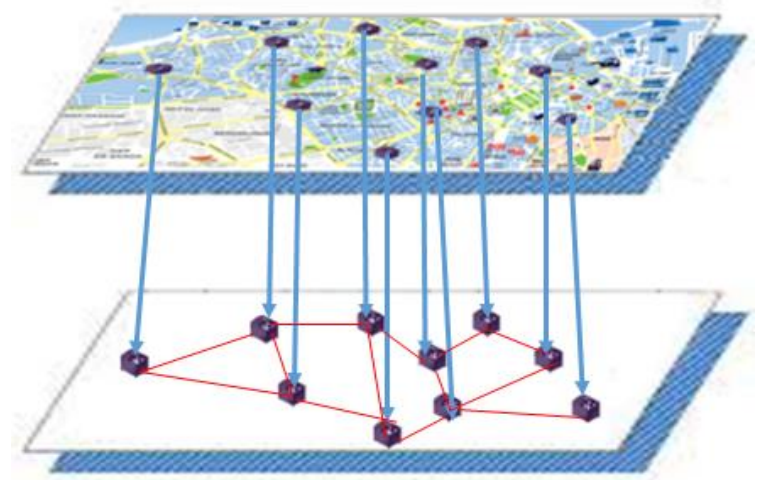

Fig. 4. Waste collection map.

\section{B. Vehicle Routing Agent (VRA)}

In order to generate the path planning for each vehicle, we are based on the Ant Colony System algorithm to build the best routes by using a set of artificial ants to find the shortest paths between the various wastes locations. The whole behavior is done by a set of distributed agents, that work in parallel to compute the best path-planning (Fig. 5) and finding the shortest path that starts from the depot and tries to visit a set of wastes stops such as each one is visited once. The VRA will evaluate the quality of each generated solution depending on the total length of each tour. The generated solution will be sent to the CCA for assigned each vehicle to the nearest tour and start the process of garbage collection, when one of those vehicles meet their capacity limit, a new request will be sent to the VRA to start again the process of creating new alternatives routes and take into consideration the last node visited by each vehicle, because each vehicle should start here tour from the last visited node and add to his pipe the new unvisited stops. 


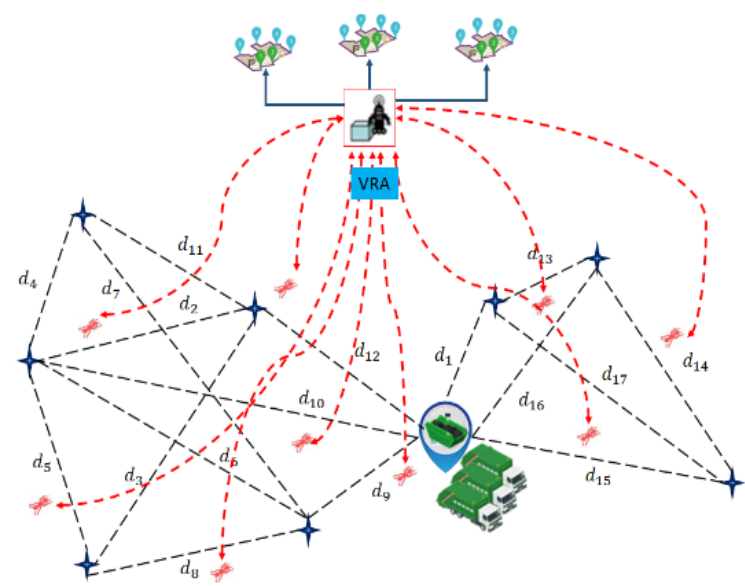

Fig. 5. Finding solution for dynamic waste collection problem.

\section{Vehicle Agent (VA)}

The Vehicle Agent (VA) is designed to guide the vehicle toward the received path planning from the CAA and control the capacity of the vehicle after each visit location. Using this information, the CCA will be able to control each vehicle and be ready to ask the VRA for some new alternatives routes in the case of exceeding the capacity limit. In parallel, the CCA informs the available VA to wait when they finish their current exploration until the new path planning is computed using the new input information prepared by the CCA.

In Fig. 6, the processes of communication between the designed agents will be based on asynchronous messages and can be modeled as follows:

- At the beginning, the user will select the wastes locations to visits and draw the available routes between those nodes; the existing vehicles will be ready to start their tours. The CCA will take the created map and initialized the parameters of our distributed system. The map will be converted to a graph with nodes (wastes locations) and arcs (routes between nodes). Therefore, we begin the process of re-routing to compute the initial path planning for each vehicle.

- The VRA will create a set of artificial ants depending on the size of the map, each agent will begin the exploration process by finding the optimal path that visit all the existing wastes with the minimum distance. The best founded solutions (the solutions with minimal distances) will be communicated with the CCA to select the best one from the existing solutions.

- Based on the proposed solutions, the CCA will associate each VA to the computed tour and allow them to start the process of collection.

- When a vehicle reaches his limit capacity, the VA sends an emergency request to the CCA, all vehicles will be waiting for the new path planning that will be created by the VRA.
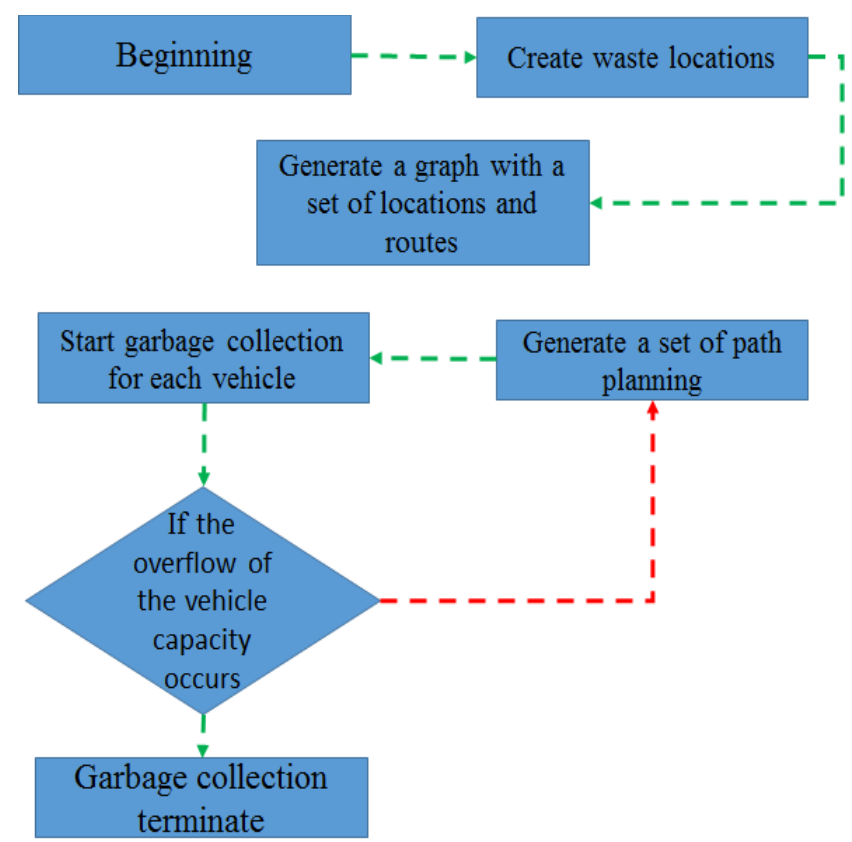

Fig. 6. Multi-agent interaction model.

\section{COMPUTATIONAL RESULTS}

In terms of solving GCS (Garbage collection system), there are several meta-heuristic methods that have been developed such as particle swarm optimization (PSO) [15], simulated annealing (SA) [16]. This paper develops a new distributed meta-heuristic method for solving GCS. It employs ACS algorithm in a distributed environment based on JADE (Java Agent Development Environment) Framework. This environment will help us to create artificial ants based on the JAVA programming language, each agent will be characterized with a specific behavior based on the equations defined in the previous section. So the returned results by each agent will be considered as a sub-solution which will be analyzed by the main agent to see the quality of the proposed solution. The classical garbage collection is clearly defined as an NP-hard combinatorial problem. Due to its complexity, many researches in this area prefers heuristic or meta-heuristic such as genetic algorithm (GA) [20], [21] rather than the exact method for solving this problem.

This section discusses a garbage collection system for a population with a limited data set. This system consists of many dump vehicles, which are available in the central depot to collect waste from several wastes stops. The volume of waste in each stop is unknown. The objective of this solution is to find the best route for vehicles to collect wastes from all locations. Theoretically, this problem can be modeled as VRP. In the practical application, this system is divided into several sub-areas, where each one has a different number of waste locations. The smallest sub-area consists of 14 stops, whereas the largest sub-system has 34 stops. 
TABLE I. COMPARISON OF THE DISTRIBUTED ACS AND GA ALGORITHM FOR SOLVING THE WCP (METERS)

\begin{tabular}{|l|l|l|l|l|l|l|}
\hline \multirow{2}{*}{$\begin{array}{l}\text { Sub- } \\
\text { area }\end{array}$} & $\begin{array}{l}\text { Nbr of } \\
\text { wastes } \\
\text { stops }\end{array}$ & $\begin{array}{l}\text { Nbr of } \\
\text { vehicles }\end{array}$ & Distributed ACO & \multicolumn{2}{|l|}{ Classical GA } \\
\cline { 4 - 7 } & & 12 & 36317 & 26652 & 27117 & 26655 \\
Bin1 & 22 & 12 Best & Average & $\begin{array}{l}\text { Best } \\
\text { sol }\end{array}$ \\
\hline Bin2 & 14 & 6 & 7321 & 7065 & 7967 & 7131 \\
\hline Bin3 & 26 & 12 & 36681 & 36425 & 39211 & 37845 \\
\hline Bin4 & 14 & 34 & 18728 & 18650 & 19079 & 18997 \\
\hline Bin5 & 34 & 22 & 24330 & 22567 & 27575 & 26557 \\
\hline
\end{tabular}

Furthermore, ACS and GA are also performed for the purpose of comparison. Computational results for these two methods are provided in Table 1. The statistical tests to evaluate if the proposed solution algorithm is significantly better than GA and executed on machine laptop HP PRO with $8 \mathrm{~GB}$ in RAM and a $2.8 \mathrm{Ghz}$ quad core processor.

In terms of computational time, the distributed solution performs relatively as fast as GA. The results show that the developed system has a better starting point than GA. This is the first advantage of the multi-agent architecture. It reveals that the initialization strategy designed for the system works successfully.

After the successful implementation of the distributed garbage collection solution for the mentioned locality, the efficiency of the designed solution has been analyzed. We are based on the total distance covered by all vehicles to visit all the available wastes stops to measure and compute the efficiency of the implementing solution. The system takes into consideration the cases when some vehicles exceed their capacity limit to see the convergence of the distributed solution in this case. The optimal solutions computed by the two methods are shown in Table 1 to see the efficiency of each method on the prepared scenarios.

\section{CONCLUSION}

A distributed waste collection vehicle routing system has been modeled in this work, which considers the re-routing of the system when one of the vehicles meet his capacity limit during the collecting process. As the problem aims to find the optimal routes between the available nodes, we are based on a new version of the Ant Colony System Algorithm to compute the best routes in terms of the total distance traveled by each vehicle and trying to minimize the cost of waste collection.

A new multi-agent ACS algorithm with artificial ants was designed for solving the presented problem. The results of two benchmark instances for garbage collection problem indicate that the best solutions have approached to the solution given by the proposed solution. The distributed technique is designed to solve the garbage collection routing problem, which aims to find the best solutions based on the ACO techniques on a distributed environment using several artificial ants, which work together in parallel to find the optimal path planning.
Future work will be done to make the developed system more flexible by supporting other constraints such as the capacity limit and introducing the notion of the vehicle routing with time windows in which the driver should visit a node at a predefined time and we study the possibility of taken into consideration the traffic flow when the system compute the best solution for the GCP.

\section{REFERENCES}

[1] Karim El Bouyahyiouy, Adil Bellabdaoui, "An ant colony optimization algorithm for solving the full truckload vehicle routing problem with profit", International Colloquium on Logistics and Supply Chain Management (LOGISTIQUA), 2017.

[2] L. Guezouli, S. Abdelhamid,"A multi-objective optimization of Multidepot Fleet Size and Mix Vehicle Routing Problem with time window", The 6th International Conference on Systems and Control (ICSC), 2017.

[3] Ying Zhou, Jiahai Wang, "A Local Search-Based Multiobjective Optimization Algorithm for Multiobjective Vehicle Routing Problem With Time Windows", IEEE Systems Journal (Volume: 9, Issue: 3, Sept. 2015).

[4] Aya Ishigaki,"An Application to Stochastic Vehicle-Routing Problem in a Waste Collection", The 5th IIAI International Congress on Advanced Applied Informatics (IIAI-AAI), 2016.

[5] Yesica Xiomara Daza Cruz, Johana Andrea Patiño Chirva, Eduyn Ramiro Lopez Santana, "A mixed integer optimization model to design a selective collection routing problem for domestic solid waste", Engineering Applications - International Congress on Engineering (WEA), 2015.

[6] Maria Pia Fanti, Agostino M. Mangini, Lorenzo Abbatecola, Walter Ukovich, "Decision support for a waste collection service with time and shift constraints", American Control Conference (ACC), 2016.

[7] Milan Miić, Aleksandar Dordevic, Aleksandra Kokić Arsić, "The optimization of vehicle routing of communal waste in an urban environment using a nearest neighbirs' algorithm and genetic algorithm: Communal waste vehicle routing optimization in urban areas",Ninth International Conference on Advanced Computational Intelligence (ICACI), 2017.

[8] Udom Janjarassuk, Ruedee Masuchun, "An ant colony optimization method for the capacitated vehicle routing problem with stochastic demands", International Computer Science and Engineering Conference (ICSEC), 2016.

[9] Xinyu Wang, Tsan-Ming Choi, Haikuo Liu, Xiaohang Yue, "Novel Ant Colony Optimization Methods for Simplifying Solution Construction in Vehicle Routing Problems", IEEE Transactions on Intelligent Transportation Systems ( Volume: 17, Issue: 11, Nov. 2016 ).

[10] Shuwei Zhang, Yugong Luo, Keqiang Li, "Multi-objective route search for electric vehicles using ant colony optimization", American Control Conference (ACC), 2016.

[11] Andrei Borozdukhin, Olga Dolinina, Vitaly Pechenkin, "Approach to the garbage collection in the Smart Clean City project", The 4th IEEE International Colloquium on Information Science and Technology (CiSt), 2016.

[12] Trushali S. Vasagade, Shabanam S. Tamboli, Archana D. Shinde, "Dynamic solid waste collection and management system based on sensors, elevator and GSM", International Conference on Inventive Communication and Computational Technologies (ICICCT), 2017.

[13] Shashika Lokuliyana, J.A.D.C. Anuradha Jayakody, Lakmal Rupasinghe, Sachini Kandawala, "IGOE IoT framework for waste collection optimization", National Conference on Technology and Management (NCTM), 2017.

[14] Md Abdulla Al Mamun, M. A. Hannan, Aini Hussain, Hassan Basri, "Wireless Sensor Network Prototype for Solid Waste Bin Monitoring with Energy Efficient Sensing Algorithm", IEEE 16th International Conference on Computational Science and Engineering (CSE), 2013

[15] Rami Abousleiman, Osamah Rawashdeh, "Electric vehicle modelling and energy-efficient routing using particle swarm optimisation", IET Intelligent Transport Systems ( Volume: 10, Issue: 2, 32016 ). 
[16] S.-W. Lin, K.-C. Ying, Z.-J. Lee, H.-S. Chen, "Vehicle Routing Problems with Time Windows Using Simulated Annealing", SMC '06. IEEE International Conference on Systems, Man and Cybernetics, 2006.

[17] Benjamin Lammel, Karin Gryzlak, Rolf Dornberger, Thomas Hanne, "An ant colony system solving the travelling salesman region problem", 4th International Symposium on Computational and Business Intelligence (ISCBI), 2016.

[18] Michalis Mavrovouniotis, Felipe M. Müller, Shengxiang Yang, "Ant Colony Optimization With Local Search for Dynamic Traveling Salesman Problems", IEEE Transactions on Cybernetics ( Volume: 47, Issue: 7, July 2017 ).

[19] Aleksandar Kaplar ; Milan Vidaković ; Nikola Luburić ; Mirjana Ivanović, "Improving a distributed agent-based Ant Colony Optimization for Solving Traveling Salesman Problem", 40th International Convention on Information and Communication Technology, Electronics and Microelectronics (MIPRO), 2017.
[20] R. J. Kuo , Ferani E. Zulvia, "Hybrid genetic ant colony optimization algorithm for capacitated vehicle routing problem with fuzzy demand A case study on garbage collection system", 4th International Conference on Industrial Engineering and Applications (ICIEA), 2017.

[21] Shijin Wang, Yulun Wu, "A genetic algorithm for energy minimization Vehicle Routing Problem", International Conference on Service Systems and Service Management (ICSSSM), 2017.

[22] M. Dorigo, L.M. Gambardella, "Ant colony system: a cooperative learning approach to the traveling salesman problem", IEEE Transactions on Evolutionary Computation (Volume: 1, Issue: 1, Apr 1997).

[23] Zulfiqar Ali, Waseem Shahzad, "Comparative Analysis and Survey of Ant Colony Optimization based Rule Miners", International Journal of Advanced Computer Science and Applications, Vol. 8, No. 1, 2017 . 